\title{
CAPACITY DISTRIBUTIONS IN SPATIAL STOCHASTIC MODELS FOR TELECOMMUNICATION NETWORKS
}

\author{
Florian Voss $^{1}$, CATHERINe Gloaguen ${ }^{2}$ And Volker SchmidT ${ }^{1}$ \\ ${ }^{1}$ Ulm University, Institute of Stochastics, Ulm, Germany; ${ }^{2}$ Orange Labs, Issy les Moulineaux, France \\ e-mail: florian.voss@uni-ulm.de \\ (Accepted September 1, 2009)
}

\begin{abstract}
We consider the stochastic subscriber line model as a spatial stochastic model for telecommunication networks and we are interested in the evaluation of the required capacities at different locations of the network in order to provide, in fine, an estimation of the cable system which has to be installed. In particular, we consider hierarchical telecommunication networks with higher-level components (HLC) and lower-level components (LLC) located on the road system underlying the network. The cable paths are modeled by shortest paths along the edge set of a stationary random tessellation, whereas both HLC and LLC are modeled by Cox processes concentrated on the edges of this tessellation. We then introduce the notion of capacity which depends on the length of some subtree on the edge set of the underlying tessellation. Moreover, we investigate estimators for the density and distribution function of the typical length of this subtree which can be computed based on Monte Carlo simulations of the typical serving zone. In a numerical study, the density of the typical subtree length is determined for different specific models.
\end{abstract}

Keywords: point processes, random tessellations, stochastic geometry, telecommunication systems.

\section{INTRODUCTION}

A statistical method based on spatial stochastic modeling is proposed in order to analyze required capacities in hierarchical telecommunication networks. The networks considered in this paper possess higher-level components (HLC) and lowerlevel components (LLC) located on the cable system of the network. With each HLC a domain is associated which is called the serving zone of this HLC. All LLC within a serving zone are then connected to its HLC on the shortest path along the road system. Recently, such telecommunication networks have been studied in the context of the stochastic subscriber line model (SSLM), where a method has been developed to estimate the mean typical shortest path length and the mean typical subscriber line length from LLC to HLC (Gloaguen et al., 2009a). Note that the SSLM is particularly suitable in the analysis of telecommunication access networks. Further methods for the analysis and optimization of telecommunication networks based on spatial stochastic models have been developed, e.g., in Baccelli and Zuyev (1996), Baccelli et al. (2000), and Baccelli and Błaszczyszyn (2001).

We investigate a network characteristic which is related to the typical shortest path length in the SSLM. In particular, we are interested in the distribution of capacities required at different locations of the network. The required capacity is an important characteristic in the strategic planning of telecommunication networks. On the one hand, it is too expensive to install cables with large capacities at all locations of the network. On the other hand, it is important that the cable system of the network provides the capacities demanded by the subscribers. However, once the network is built, it is difficult to change the installed cables of the network. So it is essential to know the required capacities at given network locations in advance. Based on our stochastic model, the approach developed in the present paper provides a method to design capacities of new networks or analyze existing cable systems.

The paper is organized as follows. First we briefly mention how the density of the typical shortest path length can be estimated, which will be used later on in the paper. Then, we introduce the notion of capacity at given network locations and show how its distribution depends on the lengths of some subtrees of the network. The considered locations are modeled by point processes, where we concentrate on two special cases. We analyze the capacity required at locations with a given shortest path length to the closest HLC and at network locations chosen at random, respectively. In both cases we derive representation formulae for distributional characteristics of the typical subtree length, which are suitable to construct estimators for these network characteristics based on samples of the typical serving zone. Finally, we present the results of a numerical study, where the density of the typical subtree length is determined for different specific models. 


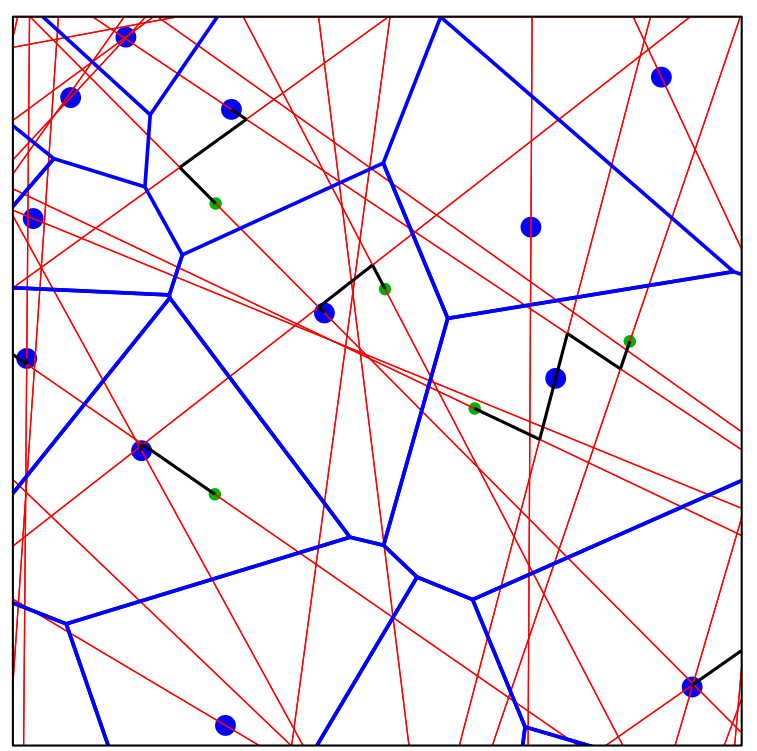

(a) PLT

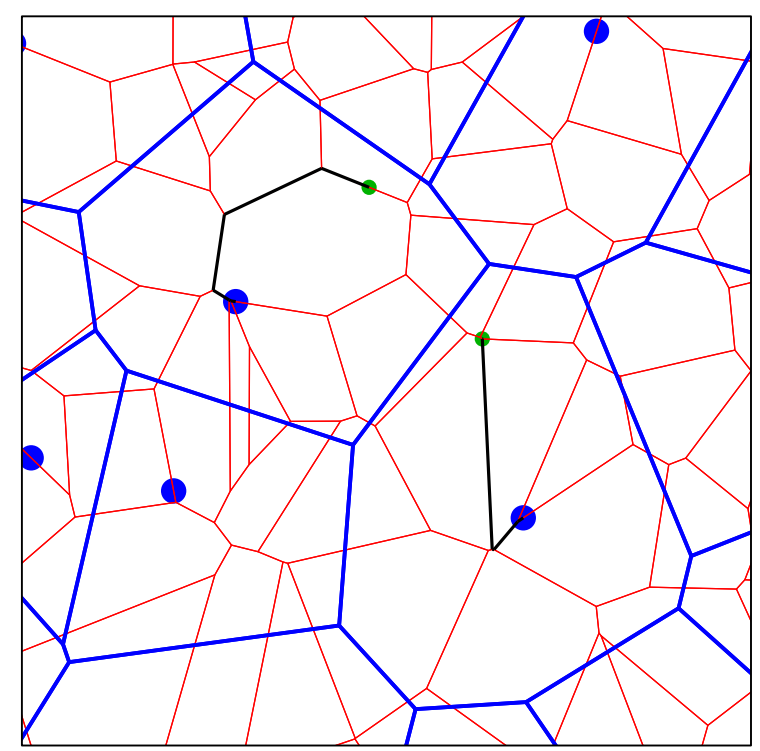

(b) PVT

Fig. 1. HLC with their serving zones (blue) and LLC (green) with shortest paths along the edge set (red).

\section{SHORTEST PATH LENGTHS}

To begin with we briefly introduce our network model which is based on (marked) point processes, random tessellations and Palm theory. For details on these basic notions from stochastic geometry, see Stoyan et al. (1995) and Schneider and Weil (2008). The cable system is modeled by the edge set $T^{(1)}$ of a stationary and isotropic random tessellation $T$ with intensity $\gamma=\mathbb{E} v_{1}\left(T^{(1)} \cap[0,1)^{2}\right)$, where $v_{1}$ denotes the 1-dimensional Hausdorff measure. For example, $T$ can be a Poisson line tessellation (PLT), a PoissonVoronoi tessellation (PVT), or a Poisson-Delaunay tessellation (PDT). The HLC and LLC are modeled as (conditionally independent) Cox processes $X_{H}$ and $X_{L}$ with linear intensities $\lambda_{\ell}$ and $\lambda_{\ell}^{\prime}$ on $T^{(1)}$, where the random driving measure $\Lambda$ of $X_{H}$ and $X_{L}$ is given by $\Lambda(B)=\tilde{\lambda} v_{1}\left(B \cap T^{(1)}\right), B \in \mathscr{B}\left(\mathbb{R}^{2}\right)$ with $\widetilde{\lambda}=\lambda_{\ell}$ and $\widetilde{\lambda}=\lambda_{\ell}^{\prime}$, respectively. Each point $X_{L, n}$ of $X_{L}$ is then connected to its nearest neighbor of $X_{H}$ with respect to the Euclidean distance. Thus $X_{L, n}$ is connected to a point $X_{H, j}$ of $X_{H}$ if and only if $X_{L, n}$ is located in the Voronoi cell $\Xi_{H, j}$ of $X_{H, j}$ induced by $X_{H}$. Furthermore, we assume that the physical connection between $X_{L, n}$ and its nearest neighbor of $X_{H}$ is obtained on the shortest path along the edge set $T^{(1)}$. However, the connection path may lie partly outside $\Xi_{H, j}$. Let $s\left(X_{L, n}\right)$ denote the length of this path and consider the stationary marked point process $X_{L}^{S}=\left\{\left(X_{L, n}, s\left(X_{L, n}\right)\right)\right\}$. The typical mark of $X_{L}^{s}$ is called the typical shortest path length. It is denoted by $S^{*}$ and can formally be defined by the Palm mark distribution of $X_{L}^{s}$. In the ergodic case, the empirical distribution of the marks $s\left(X_{L, n}\right)$ belonging to a sampling window $W$ converges to the distribution of $S^{*}$ if $W$ unboundedly increases. Thus, $S^{*}$ can be interpreted as the shortest path length of a location chosen at random among all LLC. Realizations of $X_{L}^{s}$ are displayed in Fig. 1. Let $T_{H}=\left\{\Xi_{H, n}\right\}$ denote the Voronoi tessellation of $X_{H}$ and consider the marked point process $\left\{\left(X_{H, n}, L_{H, n}^{o}\right)\right\}$, where $L_{H, n}^{o}=\left(T^{(1)} \cap \Xi_{H, n}\right)-X_{H, n}$, i.e., the points of $X_{H}$ are labelled with the (centered) segment systems of $T^{(1)}$ inside the Voronoi cells of $T_{H}$. The segment system $L_{H}^{*}$ inside the typical cell of $T_{H}$ is then defined as the typical mark of $\left\{\left(X_{H, n}, L_{H, n}^{o}\right)\right\}$. Note that $L_{H}^{*}$ can be split into segments $S_{i}, i=1, \ldots, M$ with endpoints $A_{i}, B_{i}$ such that $s\left(A_{i}\right)<s\left(B_{i}\right)=s\left(A_{i}\right)+v_{1}\left(S_{i}\right)$, where $s(x)$ denotes the length of the shortest path from $x \in L_{H}^{*}$ to the origin, see Fig. 2.

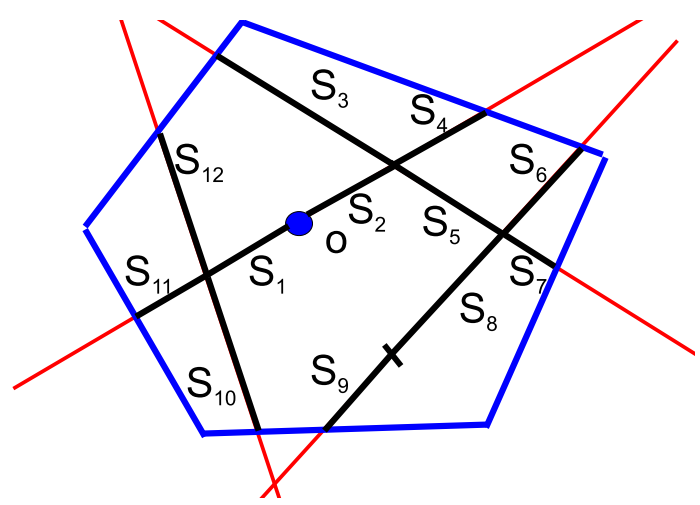

Fig. 2. Splitting of $L_{H}^{*}$ into segments $S_{1}, \ldots, S_{M}$.

Using Neveu's exchange formula for stationary marked point processes, the following representation 
formula can be derived (Gloaguen et al., 2009b; Voss et al., 2009c).

Lemma 1 The density $f_{S^{*}}$ of $S^{*}$ is given by

$$
f_{S^{*}}(x)= \begin{cases}\lambda_{\ell} \mathbb{E}\left[\sum_{i=1}^{M} \mathbb{I}_{\left[s\left(A_{i}\right), s\left(B_{i}\right)\right)}(x)\right] & \text { if } x \geq 0, \\ 0 & \text { otherwise, }\end{cases}
$$

where $\mathbb{I}_{\left[s\left(A_{i}\right), s\left(B_{i}\right)\right)}(x)$ equals 1 if $x \in\left[s\left(A_{i}\right), s\left(B_{i}\right)\right)$ and 0 otherwise.

Note that the density $f_{S^{*}}$ of $S^{*}$ does not depend on the intensity $\lambda_{\ell}^{\prime}$ of LLC. Furthermore, the representation formula given in Lemma 1 leads to the (plug-in) estimator

$$
\widehat{f}_{S^{*}}(x ; n)=\lambda_{\ell} \frac{1}{n} \sum_{j=1}^{n} \sum_{i=1}^{M_{j}} \mathbb{I}_{\left[s\left(A_{i, j}\right), s\left(B_{i, j}\right)\right)}(x),
$$

for $f_{S^{*}}(x)$ which can be computed based on the simulation of $n$ i.i.d. copies $L_{H, 1}^{*}, \ldots, L_{H, n}^{*}$ of $L_{H}^{*}$. For $T$ being a PLT, PVT, and PDT, respectively, simulation algorithms for $L_{H}^{*}$ are given in Gloaguen et al. (2005), Fleischer et al. (2009), and Voss et al. (2009a). Note that the estimator $\widehat{f}_{S^{*}}(\cdot ; n)$ given in Eq. 1 has good statistical properties. For example, it can be shown that $\mathbb{P}\left(\lim _{n \rightarrow \infty} \sup _{x \in \mathbb{R}}\left|\widehat{f}_{S^{*}}(x ; n)-f_{S^{*}}(x)\right|=0\right)=1$, see Voss et al. $(2009 \mathrm{c})$.

\section{MODELING OF CAPACITIES}

The main topic of this paper is the modeling and analysis of capacities required at various locations in telecommunication networks. They are important performance characteristics and should be investigated before the networks are built physically, because it should be guaranteed as a rule that the capacity at a given location is higher than the demand at this location. Otherwise, the demand of some LLC cannot be served. Thus, for strategic planning of telecommunication networks, the analysis of capacities required at given locations of the network is an important task.

More formally, the capacity required at a given point $x \in T^{(1)}$ of the edge set $T^{(1)}$ is understood as the sum of demands which are requested by all those LLC located in the same serving zone as $x$ and whose shortest paths cross $x$. In connection with this, we consider a sequence of independent and identically distributed random variables $C_{1}, C_{2}, \ldots$ which describe the demand of the LLC at the locations $X_{L, 1}, X_{L, 2}, \ldots$, respectively. Moreover, let $X=\left\{X_{n}\right\}$ be a further stationary point process of random locations $X_{n}$ on the edge set $T^{(1)}$ which is conditionally independent of $X_{L}$ given $T^{(1)}$. The points of $X$ are used to model the locations at which we want to analyze the required capacities. The (planar) intensity of $\left\{X_{n}\right\}$ is denoted by $\lambda$. For each $n \geq 1$, we define the capacity $C\left(X_{n}\right)$ required at $X_{n}$ in the following way. If $X_{n} \in L_{H, j}=$ $L_{H, j}^{o}+X_{H, j}$ for some $j \geq 1$, then we put

$$
C\left(X_{n}\right)=\sum_{i=1}^{\infty} \mathbb{I}_{T_{\text {sub }}\left(X_{n}\right)}\left(X_{L, i}\right) C_{i},
$$

where $T_{\text {sub }}\left(X_{n}\right)=\left\{y \in L_{H, j}: X_{n} \in P\left(y, X_{H, j}\right)\right\}$ is the subset of those points $y$ on $L_{H, j}$ whose shortest path $P\left(y, X_{H, j}\right)$ from $y$ to $X_{H, j}$ crosses $X_{n}$, see Fig. 3. Note that we call $T_{\text {sub }}\left(X_{n}\right)$ the subtree rooted at $X_{n}$.

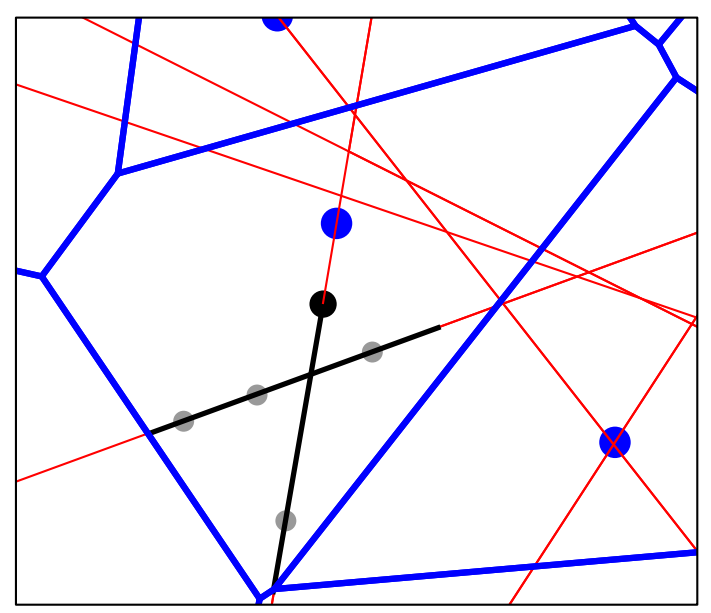

Fig. 3. $T_{\text {sub }}(x)$ (black) at given location $x$ (black) with LLC (grey) on subtree.

Since $X_{n}$ is conditionally independent of $X_{L}$ given $T^{(1)}$, Eq. 2 implies that $C\left(X_{n}\right) \stackrel{\mathrm{d}}{=} \sum_{i=1}^{K_{n}} C_{i}$, where $K_{n} \sim \operatorname{Poi}\left(\lambda_{\ell}^{\prime} v_{1}\left(T_{\text {sub }}\left(X_{n}\right)\right)\right)$ given $v_{1}\left(T_{\text {sub }}\left(X_{n}\right)\right)$. Here $\operatorname{Poi}(\mu)$ denotes the Poisson distribution with mean $\mu$. Thus, the distribution of $C\left(X_{n}\right)$ is fully characterized by $\lambda_{\ell}^{\prime}$ and the distribution of $C_{1}$ and the subtree length $v_{1}\left(T_{\text {sub }}\left(X_{n}\right)\right)$, respectively. Moreover, a similar representation formula can be derived for the typical capacity $C^{*}$ (at the typical point of $\left\{X_{n}\right\}$ ), where the nonnegative random variable $C^{*}$ is distributed according to the Palm mark distribution of the stationary marked point process $\left\{\left(X_{n}, C\left(X_{n}\right)\right)\right\}$.

Theorem 1 It holds that

$$
C^{*}=\sum_{i=1}^{K^{*}} C_{i}
$$

where $K^{*} \sim \operatorname{Poi}\left(\lambda_{\ell}^{\prime} v_{1}\left(T_{\text {sub }}^{*}\right)\right)$ given $v_{1}\left(T_{\text {sub }}^{*}\right)$ and the random variable $v_{1}\left(T_{\text {sub }}^{*}\right)$ is distributed according to the Palm mark distribution of $X^{T}=$ $\left\{\left(X_{n}, v_{1}\left(T_{\text {sub }}\left(X_{n}\right)\right)\right)\right\}$. 
Proof Using Eq. 2, the definition of the (Palm mark) distribution of $C^{*}$ gives that for each $h: \mathbb{R} \rightarrow[0, \infty)$

$$
\begin{aligned}
& \mathbb{E} h\left(C^{*}\right)=\frac{1}{\lambda} \mathbb{E}\left(\sum_{X_{i} \in[0,1]^{2}} h\left(\sum_{X_{L, j} \in T_{\text {sub }}\left(X_{i}\right)} C_{j}\right)\right) \\
& =\frac{1}{\lambda} \mathbb{E}\left[\mathbb{E}\left(\sum_{X_{i} \in[0,1]^{2}} h\left(\sum_{X_{L, j} \in T_{\text {sub }}\left(X_{i}\right)} C_{j}\right) \mid T^{(1)}, X_{H}\right)\right],
\end{aligned}
$$

where

$$
\begin{aligned}
\mathbb{E}\left(\sum_{i} h\left(\sum_{j} C_{j}\right) \mid T^{(1)}, X_{H}\right) \\
=\mathbb{E}\left[\mathbb{E}\left(\sum_{i} h\left(\sum_{j} C_{j}\right) \mid X\right) \mid T^{(1)}, X_{H}\right] \\
=\mathbb{E}\left[\sum_{i} \mathbb{E}\left(h\left(\sum_{j} C_{j}\right) \mid X\right) \mid T^{(1)}, X_{H}\right]
\end{aligned}
$$

and

$$
\mathbb{E}\left(h\left(\sum_{j} C_{j}\right) \mid X\right)=\mathbb{E}\left(h\left(\sum_{j=1}^{K_{i}} C_{j}\right) \mid v_{1}\left(T_{\mathrm{sub}}\left(X_{i}\right)\right)\right.
$$

with $K_{i} \sim \operatorname{Poi}\left(\lambda_{\ell}^{\prime} v_{1}\left(T_{\text {sub }}\left(X_{i}\right)\right)\right)$ given $v_{1}\left(T_{\text {sub }}\left(X_{i}\right)\right)$. Thus, the statement of the theorem follows from the definition of the Palm mark distribution of the stationary marked point process $X^{T}=$ $\left\{\left(X_{n}, v_{1}\left(T_{\text {sub }}\left(X_{n}\right)\right)\right)\right\}$.

In view of Eq. 3, it suffices to investigate the distribution of the typical subtree length $v_{1}\left(T_{\text {sub }}^{*}\right)$, assuming that $\lambda_{\ell}^{\prime}$ and the distribution of $C_{1}$ are known. Similar to the statement of Lemma 1, we can express the distribution of $v_{1}\left(T_{\mathrm{sub}}^{*}\right)$ in terms of the typical segment system $L_{H}^{*}$, using again Neveu's exchange formula. Let $\lambda_{H}$ denote the (planar) intensity of $X_{H}$ and let $\widetilde{X}^{T}$ be the Palm version of $X^{T}$ which is obtained under the Palm probability measure of $\left\{\left(X_{H, n}, L_{H, n}^{o}\right)\right\}$ (see, e.g., Fleischer et al., 2009 for details). Then, the following is true.

Theorem 2 For any measurable $h:[0, \infty) \rightarrow[0, \infty)$,

$$
\mathbb{E} h\left(v_{1}\left(T_{\mathrm{sub}}^{*}\right)\right)=\frac{\lambda_{H}}{\lambda} \mathbb{E} \int_{L_{H}^{*} \times[0, \infty)} h(y) \widetilde{X}^{T}(\mathrm{~d}(x, y)) .
$$

Proof Consider the two jointly stationary marked point processes $X^{H}=\left\{\left(X_{H, n}, L_{H, n}^{o}\right)\right\}$ and $X^{T}$. We can regard $\left(X^{T}, X^{H}\right)$ as a random element of the space $N_{[0, \infty), \mathscr{L}}$ of locally finite point sets with marks in $[0, \infty)$ or $\mathscr{L}$, where $\mathscr{L}$ denotes the family of all locally finite segment systems containing the origin. Using the function $f: \mathbb{R}^{2} \times[0, \infty) \times \mathscr{L} \times N_{[0, \infty), \mathscr{L}} \rightarrow[0, \infty)$ given by

$$
f(x, s, \zeta, \psi)= \begin{cases}h(s) & \text { if } x \in \zeta, \\ 0 & \text { otherwise }\end{cases}
$$

we can apply Neveu's exchange formula (Neveu, 1976; Maier et al., 2004) for stationary marked point processes which yields Eq. 4.

In the rest of this paper we focus on two special cases for the point process $\left\{X_{n}\right\}$ of locations at which the required capacities are considered.

\section{FIXED DISTANCE TO HLC}

For some $s>0$, let $X=\left\{X_{n}\right\}$ be the point process which consists of all points of $\left\{x \in T^{(1)}: s(x)=s\right\}$, where $s(x)$ denotes the shortest path length from $x$ to $X_{H, j}$ if $x \in L_{H, j}$, i.e., $X$ is the point process of those points on $T^{(1)}$ with a fixed shortest path length to their HLC which is equal to $s$. Then, the statement of Theorem 2 can be specified in the following way.

Theorem 3 For any measurable $h:[0, \infty) \rightarrow[0, \infty)$,

$$
\mathbb{E} h\left(v_{1}\left(T_{\text {sub }}^{*}\right)\right)=\frac{\lambda_{\ell}}{f_{S^{*}}(s)} \mathbb{E} \sum_{i=1}^{\widetilde{K}} h\left(v_{1}\left(T_{\text {sub }}\left(\widetilde{X}_{i}\right)\right)\right),
$$

where $\left(\widetilde{X}_{1}, T_{\text {sub }}\left(\widetilde{X}_{1}\right)\right), \ldots,\left(\widetilde{X}_{\widetilde{K}}, T_{\text {sub }}\left(\widetilde{X}_{\widetilde{K}}\right)\right)$ denote the marked points of $\widetilde{X}^{T}$ on $L_{H}^{*}$ and $\widetilde{K}$ is (random) total number of these points. In particular, the distribution function $F: \mathbb{R} \rightarrow[0,1]$ of $v_{1}\left(T_{\mathrm{sub}}^{*}\right)$ is given by

$$
F(x)=\frac{\lambda_{\ell}}{f_{S^{*}}(s)} \mathbb{E} \sum_{i=1}^{\widetilde{K}} \mathbb{I}_{[0, x]}\left(v_{1}\left(T_{\text {sub }}\left(\widetilde{X}_{i}\right)\right)\right), \quad x \geq 0 .
$$

Proof Using Eq. 4 we only have to show that $\lambda_{H} / \lambda=$ $\lambda_{\ell} / f_{S^{*}}(s)$. Moreover, since $\lambda_{H}=\gamma \lambda_{\ell}$, it suffices to show that $\lambda=\gamma f_{S^{*}}(s)$. We have that

$$
\begin{aligned}
\lambda & =\mathbb{E} \#\left\{n: X_{n} \in[0,1)^{2}\right\} \\
& =\mathbb{E} \sum_{i=1}^{\infty} \#\left\{n: X_{n} \in L_{H, i} \cap[0,1)^{2}\right\} \\
& =\lambda_{H} \int_{\mathbb{R}^{2}} \mathbb{E} \#\left\{n: \widetilde{X}_{n} \in L_{H}^{*} \cap\left([0,1)^{2}-x\right)\right\} \mathrm{d} x,
\end{aligned}
$$

where the latter equality follows from the refined Campbell theorem for stationary marked point processes. Note that each point $\widetilde{X}_{i}, i=1, \ldots, \widetilde{K}$ is contained almost surely in exactly one segment of $L_{H}^{*}$ and each segment contains 0 or 1 points of $\widetilde{X}^{T}$, so $\widetilde{K} \leq M$. We can assume without loss of generality that $\widetilde{X}_{i} \in S_{i}$ for $i=1, \ldots, \widetilde{K}$ and that the segments 
$S_{i}, i=\widetilde{K}+1, \ldots, M$ do not contain a point with shortest path length $s$. This yields

$$
\begin{aligned}
\lambda & =\lambda_{H} \int_{\mathbb{R}^{2}} \mathbb{E} \#\left\{n: \widetilde{X}_{n} \in L_{H}^{*} \cap\left([0,1)^{2}-x\right)\right\} \mathrm{d} x \\
& =\gamma \lambda_{\ell} \mathbb{E} \sum_{j=1}^{\widetilde{K}} \mathbb{I}_{\left[s\left(A_{j}\right), s\left(B_{j}\right)\right)}(s) \int_{\mathbb{R}^{2}} \mathbb{I}_{[0,1)^{2}-\widetilde{X}_{j}}(x) \mathrm{d} x \\
& =\gamma \lambda_{\ell} \mathbb{E} \sum_{j=1}^{\widetilde{K}} \mathbb{I}_{\left[s\left(A_{j}\right), s\left(B_{j}\right)\right)}(s)=\gamma f_{S^{*}}(s),
\end{aligned}
$$

where the latter equality follows from Lemma 1. This completes the proof.

Note that $f_{S^{*}}(s)$ is not known analytically, but it can be estimated consistently by the estimator $\widehat{f}_{S^{*}}(x ; n)$ given in Eq. 1. Thus, Theorem 3 leads to a natural estimator for $F(x)$ which is given by

$$
\widehat{F}(x ; n)=\frac{\lambda_{\ell}}{\widehat{f}_{S^{*}}(x ; n)} \frac{1}{n} \sum_{j=1}^{n} \sum_{i=1}^{\widetilde{K}^{(j)}} \mathbb{I}_{[0, x]}\left(v_{1}\left(T_{\mathrm{sub}}^{(j)}\left(\widetilde{X}_{i}^{(j)}\right)\right)\right)
$$

where $\widetilde{K}^{(j)}$ and $T_{\text {sub }}^{(j)}\left(\widetilde{X}_{i}^{(j)}\right), j=1, \ldots, n$ are i.i.d. copies of $\widetilde{K}$ and $v_{1}\left(T_{\text {sub }}\left(\widetilde{X}_{i}\right)\right)$, respectively. It is easy to see that $\widehat{F}(x ; n)$ is ratio-unbiased and strongly consistent for $F(x)$. Moreover, since $F$ is continuous, it can be easily shown that

$$
\mathbb{P}\left(\lim _{n \rightarrow \infty} \sup _{x \in \mathbb{R}}|\widehat{F}(x ; n)-F(x)|=0\right)=1 .
$$

\section{RANDOM LOCATIONS}

In this section we assume that $X=\left\{X_{n}\right\}$ is a Cox process with linear intensity $\lambda_{\ell}^{\prime \prime}=\lambda / \gamma$ on $T^{(1)}$, which is conditionally independent of $X_{H}$ and $X_{L}$, given $T^{(1)}$. Recall that $L_{H}^{*}$ can be split into segments $S_{i}, i=1, \ldots, M$ with endpoints $A_{i}, B_{i}$ such that $s\left(A_{i}\right)<$ $s\left(B_{i}\right)=s\left(A_{i}\right)+v_{1}\left(S_{i}\right)$. This leads to the following representation formula for the density $f: \mathbb{R} \rightarrow[0, \infty)$ of the typical subtree length $v_{1}\left(T_{\text {sub }}^{*}\right)$, which is similar to the formula stated in Lemma 1 for the density $f_{S^{*}}$ of $S^{*}$.

\section{Theorem 4 It holds that}

$$
f(x)= \begin{cases}\lambda_{\ell} \mathbb{E}\left[\sum_{i=1}^{M} \mathbb{I}_{\left[l\left(B_{i}\right), l\left(A_{i}\right)\right)}(x)\right] & \text { if } x \geq 0, \\ 0 & \text { otherwise }\end{cases}
$$

where $l\left(B_{i}\right)$ denotes the subtree length at $B_{i} \in L_{H}^{*}$ and $l\left(A_{i}\right)=v_{1}\left(S_{i}\right)+l\left(B_{i}\right)$.
Note that Theorem 4 can be proven directly using Theorem 2 and the assumption that $\left\{X_{n}\right\}$ is a Cox process on $T^{(1)}$. However, interestingly enough, there is an alternative proof which uses the following relationship between the distribution of the typical subtree length $v_{1}\left(T_{\text {sub }}^{*}\right)$ at the points of $\left\{X_{n}\right\}$ and the typical subtree length at the locations of the point process $\left\{X_{s, n}\right\}$ with fixed shortest path length $s$ considered in the preceding section.

Lemma 2 Let $s \in[0, \infty)$, then it holds that

$$
\mathbb{P}\left(v_{1}\left(T_{\text {sub }}^{*}\right) \leq x \mid S_{X}^{*}=s\right)=F_{S}(x),
$$

where $S_{X}^{*}$ denotes the shortest path length at the typical point of $X$ and $F_{S}$ denotes the distribution function introduced in Eq. 7 for fixed shortest path length s.

Proof Let $\widetilde{K}_{s}$ and $\widetilde{X}_{s, 1}, \ldots, \widetilde{X}_{s, \widetilde{K}_{s}}$ be the random variables which have been introduced in Theorem 3 for fixed $s \in[0, \infty)$, i.e., $s\left(\widetilde{X}_{s, i}\right)=s$ for $i=1, \ldots, \widetilde{K}_{s}$, where $\widetilde{K}_{s}$ is the number of points on $L_{H}^{*}$ with shortest path length $s$ to the origin. Moreover, let $\widetilde{X}=\left\{\widetilde{X}_{n}\right\}$ denote the Palm version of $X$ with respect to the Palm distribution of $X_{H}$. Since the density $f_{S^{*}}(s)$ does not depend on the intensity $\lambda_{\ell}^{\prime}$ of the underlying Cox process $\left\{X_{L, n}\right\}$ of LLC, see Lemma 1, the equality $f_{S^{*}}(s)=f_{S_{X}^{*}}(s)$ holds almost everywhere. Thus we have

$$
\begin{aligned}
& \mathbb{P}\left(v_{1}\left(T_{\mathrm{sub}}^{*}\right) \leq x \mid S_{X}^{*}=s\right) \\
& =\lim _{\varepsilon \searrow 0} \mathbb{P}\left(v_{1}\left(T_{\mathrm{sub}}^{*}\right) \leq x \mid S_{X}^{*} \in[s, s+\varepsilon)\right) \\
& =\lim _{\varepsilon \searrow 0} \mathbb{E}\left(\mathbb{I}_{[0, x]}\left(v_{1}\left(T_{\mathrm{sub}}^{*}\right)\right) \frac{\mathbb{I}_{[s, s+\varepsilon)}\left(S_{X}^{*}\right)}{\int_{s}^{s+\varepsilon} f_{S_{X}^{*}}(u) \mathrm{d} u}\right) \\
& =\lim _{\varepsilon \backslash 0} \frac{\lambda_{\ell}}{\lambda_{\ell}^{\prime \prime}} \mathbb{E} \sum_{\widetilde{X}_{n} \in L_{H}^{*}} \mathbb{I}_{[0, x]}\left(v_{1}\left(T_{\mathrm{sub}}\left(\widetilde{X}_{n}\right)\right)\right) \frac{\mathbb{I}_{[s, s+\varepsilon)}\left(s\left(\widetilde{X}_{n}\right)\right)}{\int_{s}^{s+\varepsilon} f_{S^{*}}(u) \mathrm{d} u} \\
& =\lambda_{\ell} \lim _{\varepsilon \backslash 0} \mathbb{E} \int_{L_{H}^{*}} \mathbb{I}_{[0, x]}\left(v_{1}\left(T_{\mathrm{sub}}(y)\right)\right) \frac{\mathbb{I}_{[s, s+\varepsilon)}(s(y))}{\int_{s}^{s+\varepsilon} f_{S^{*}}(u) \mathrm{d} u} v_{1}(\mathrm{~d} y),
\end{aligned}
$$

where the last but one equality is obtained by a slight modification of Theorem 2 and the last equality follows from the fact that the points $\left\{\widetilde{X}_{n}\right\}$ form a Cox process on $L_{H}^{*}$ with linear intensity $\lambda_{\ell}^{\prime \prime}$, see Fleischer et al. (2009). We can divide $L_{H}^{*}$ into the segments $S_{1}, \ldots, S_{M}$ as before and get

$$
\begin{aligned}
& \mathbb{P}\left(v_{1}\left(T_{\text {sub }}^{*}\right) \leq x \mid S_{X}^{*}=s\right) \\
& =\lambda_{\ell} \lim _{\varepsilon \searrow 0} \mathbb{E} \sum_{i=1}^{M} \int_{S_{i}} \mathbb{I}_{[0, x]}\left(v_{1}\left(T_{\text {sub }}(y)\right)\right) \frac{\mathbb{I}_{[s, s+\varepsilon)}(s(y))}{\int_{s}^{s+\varepsilon} f_{S^{*}}(u) \mathrm{d} u} v_{1}(\mathrm{~d} y) .
\end{aligned}
$$


Furthermore, $f_{S^{*}}$ is right-continuous, thus we have almost surely

$$
\begin{aligned}
\lim _{\varepsilon \searrow 0} & \sum_{i=1}^{M} \int_{S_{i}} \mathbb{I}_{[0, x]}\left(v_{1}\left(T_{\text {sub }}(y)\right)\right) \frac{\mathbb{I}_{[s, s+\varepsilon)}(s(y))}{\int_{s}^{s+\varepsilon} f_{S^{*}}(u) \mathrm{d} u} v_{1}(\mathrm{~d} y) \\
= & \frac{1}{f_{S^{*}}(s)} \sum_{i=1}^{\widetilde{K}_{s}} \mathbb{I}_{[0, x]}\left(v_{1}\left(T_{\mathrm{sub}}\left(\widetilde{X}_{s, i}\right)\right)\right) .
\end{aligned}
$$

Since

$$
\begin{aligned}
\int_{S_{i}} \mathbb{I}_{[0, x]}\left(v_{1}\left(T_{\mathrm{sub}}(y)\right)\right) & \frac{\mathbb{I}_{[s, s+\varepsilon)}(s(y))}{\int_{s}^{s+\varepsilon} f_{S^{*}}(u) \mathrm{d} u} v_{1}(\mathrm{~d} y) \\
\leq & \int_{S_{i}} \frac{\mathbb{I}_{[s, s+\varepsilon)}(s(y))}{\varepsilon \min _{x \in[s, s+1)} f_{S^{*}}(x)} v_{1}(\mathrm{~d} y) \\
& \leq \frac{1}{\min _{x \in[s, s+1)} f_{S^{*}}(x)}<\infty,
\end{aligned}
$$

we can use the dominated convergence theorem in order to get

$$
\begin{aligned}
& \mathbb{P}\left(v_{1}\left(T_{\mathrm{sub}}^{*}\right) \leq x \mid S_{X}^{*}=s\right) \\
& \quad=\frac{\lambda_{\ell}}{f_{S^{*}}(s)} \mathbb{E} \sum_{i=1}^{\widetilde{K}_{s}} \mathbb{I}_{[0, x]}\left(T_{\mathrm{sub}}\left(\widetilde{X}_{s, i}\right)\right)=F_{S}(x),
\end{aligned}
$$

which completes the proof.

Note that Lemma 2 states that the distribution function $F_{S}$ can be regarded as the conditional distribution function of $v_{1}\left(T_{\text {sub }}^{*}\right)$ given that $S_{X}^{*}=s$. We now use this relationship in order to prove Theorem 4.

Proof of Theorem 4 For each $x \geq 0$ Lemma 2 yields

$$
\begin{aligned}
F(x) & =\mathbb{E}\left(\mathbb{P}\left(v_{1}\left(T_{\text {sub }}^{*}\right) \leq x \mid S_{X}^{*}\right)\right) \\
& =\int_{0}^{\infty} \mathbb{P}\left(v_{1}\left(T_{\text {sub }}^{*}\right) \leq x \mid S_{X}^{*}=s\right) f_{S_{X}^{*}}(s) \mathrm{d} s \\
& =\int_{0}^{\infty} F_{S}(x) f_{S_{X}^{*}}(s) \mathrm{d} s \\
& =\int_{0}^{\infty} \frac{\lambda_{\ell}}{f_{S^{*}}(s)} \mathbb{E} \sum_{i=1}^{\widetilde{K}_{s}} \mathbb{I}_{[0, x]}\left(T_{\text {sub }}\left(\widetilde{X}_{s, i}\right)\right) f_{S_{X}^{*}}(s) \mathrm{d} s,
\end{aligned}
$$

where the latter equality follows from Eq. 7 and $\widetilde{K}_{s}, \widetilde{X}_{s, 1}, \ldots, \widetilde{X}_{s, \widetilde{K}_{s}}$ are the random variables introduced in the proof of Lemma 2. Since $f_{S^{*}}(s)=f_{S_{X}^{*}}(s)$ for almost all $s \in[0, \infty)$, we have

$$
\begin{aligned}
F(x) & =\lambda_{\ell} \mathbb{E} \int_{0}^{\infty} \sum_{i=1}^{\widetilde{K}_{s}} \mathbb{I}_{[0, x]}\left(T_{\text {sub }}\left(\widetilde{X}_{s, i}\right)\right) \mathrm{d} s \\
& =\lambda_{\ell} \mathbb{E} \sum_{i=1}^{M} \int_{l\left(B_{i}\right)}^{l\left(A_{i}\right)} \mathbb{I}_{[0, x]}(s) \mathrm{d} s \\
& =\int_{0}^{x} \lambda_{\ell} \mathbb{E} \sum_{i=1}^{M} \mathbb{I}_{\left[l\left(B_{i}\right), l\left(A_{i}\right)\right)}(s) \mathrm{d} s,
\end{aligned}
$$

which proves the theorem.

For each $x \geq 0$, Theorem 4 leads to the natural estimator

$$
\widehat{f}(x ; n)=\lambda_{\ell} \frac{1}{n} \sum_{j=1}^{n} \sum_{i=1}^{M^{(j)}} \mathbb{I}_{\left[l\left(B_{i}^{(j)}\right), l\left(A_{i}^{(j)}\right)\right)}(x),
$$

for $f(x)$ which is based on $n$ independent and identically distributed copies of $L_{H}^{*}$. Clearly, $\widehat{f}(x ; n)$ is unbiased and strongly consistent for $f(x)$. For further useful properties of $\widehat{f}(x ; n)$, see Voss et al. (2009c).

\section{NUMERICAL RESULTS}

We now consider two specific examples assuming that $T$ is a PLT or PVT, respectively. Then, it is not difficult to show that the distribution function $F$ of the typical subtree length $v_{1}\left(T_{\text {sub }}^{*}\right)$, considered in Eq. 7 for network locations with a fixed distance to their HLC, has a density. To see this, suppose that the typical segment system $L_{H}^{*}$ and the marked points $\left(\widetilde{X}_{1}, T_{\text {sub }}\left(\widetilde{X}_{1}\right)\right), \ldots,\left(\widetilde{X}_{\widetilde{K}}, T_{\text {sub }}\left(\widetilde{X}_{\widetilde{K}}\right)\right)$ as well as the random tessellation $T^{*}$ with respect to the Palm distribution of $X_{H}$ are given. If we only condition on $T^{*}$, the distribution of $\sum_{i=1}^{\widetilde{K}} \mathbb{I}_{B}\left(v_{1}\left(T_{\text {sub }}\left(\widetilde{X}_{i}\right)\right)\right)$ does not change if each HLC is replaced by a new HLC which is uniformly distributed on the same segment. Under this transformation the points with shortest path length $s$ on $T^{*}$ are not changed, but some new points may lie on $L_{H}^{*}$ and some points may not lie on $L_{H}^{*}$ anymore. However, the subtree lengths $T_{\text {sub }}\left(\widetilde{X}_{i}\right), i=1,2, \ldots$ are changed in a continuous and non-constant way with probability one if HLC are shifted along the segments. Thus $\sum_{i=1}^{\widetilde{K}} \mathbb{I}_{B}\left(v_{1}\left(T_{\text {sub }}\left(\widetilde{X}_{i}\right)\right)\right)=0$ almost surely if $v_{1}(B)=0$ and hence the distribution of $v_{1}\left(T_{\text {sub }}^{*}\right)$ is absolutely continuous.

Moreover, a scaling invariance can be observed if the scaling factor $\kappa=\gamma / \lambda_{\ell}$ is fixed, i.e., the structure of the network model is fixed, but on different scales (Gloaguen et al., 2009b; Voss et al., 2009a). We thus used the estimators introduced in the preceding sections to determine the density $f(x)$ of the typical subtree length $v_{1}\left(T_{\text {sub }}^{*}\right)$ for different values of $\kappa$ based on i.i.d. samples of $L_{H}^{*}$ which were generated using simulation algorithms introduced in Gloaguen et al. (2005) and Fleischer et al. (2009). For each realization of $L_{H}^{*}$ first the shortest path from $o$ to all nodes of $L_{H}^{*}$ were computed using Dijkstra's algorithm (Dijkstra, 1959). In a second step, segments with distance peak were split and in this way $L_{H}^{*}$ was transformed into a tree structure, the shortest path tree. Note that the distance peaks are the leaves of the tree. Based on this 


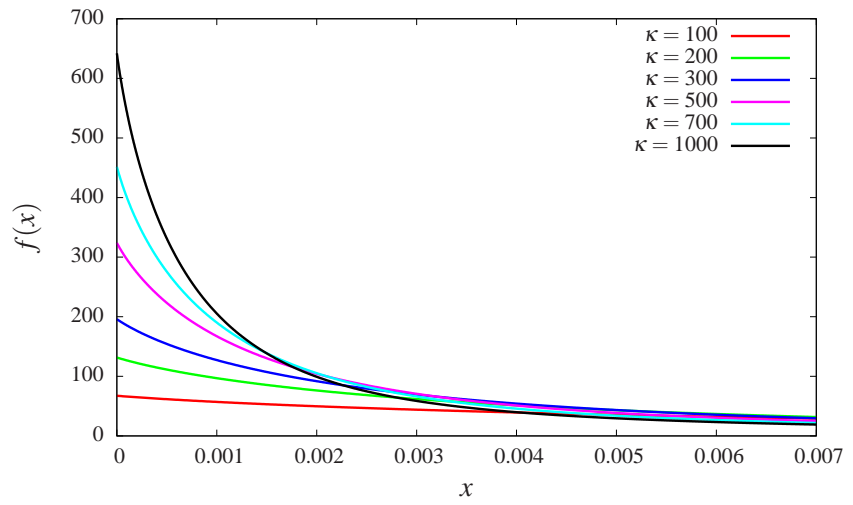

(a) PLT

Fig. 4. Density fof $v_{1}\left(T_{\text {sub }}^{*}\right)$ for Cox processes $\left\{X_{n}\right\}$.

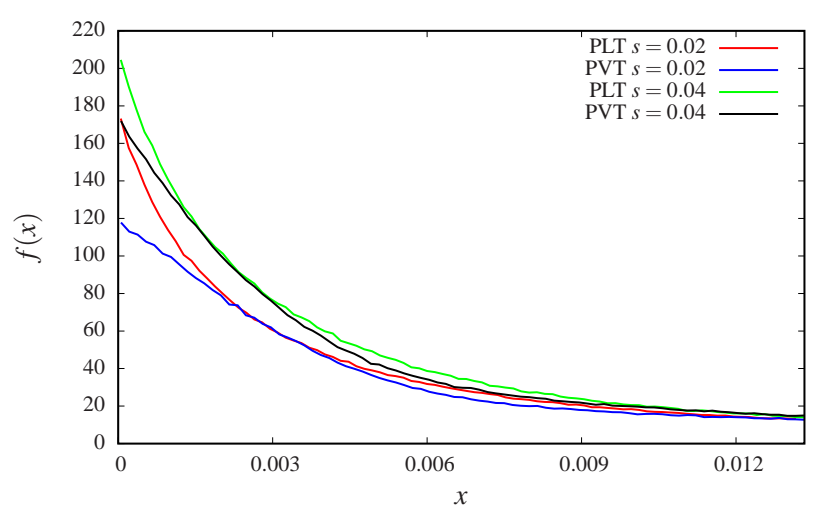

(a) $\kappa=300$

Fig. 5. Density $f$ of $v_{1}\left(T_{\text {sub }}^{*}\right)$ for fixed distance s to HLC.

tree we computed the subtree length $l\left(B_{i}\right)$ and $l\left(A_{i}\right)$ at the segment endpoints $A_{i}, B_{i}, i=1, \ldots, M$. These results were then directly used for the computation of the estimator $\widehat{f}(x ; n)$ in Eq. 10. In order to compute the estimator $\widehat{F}_{s}(x ; n)$ in Eq. 8 we first chose the segments $S_{i}, i=1, \ldots, \widetilde{K}$ with $s\left(A_{i}\right) \leq s<s\left(B_{i}\right)$, i.e., the segments which contain the points $\widetilde{X}_{1}, \ldots, \widetilde{X}_{\widetilde{K}}$, and computed $v_{1}\left(T_{\text {sub }}\left(\widetilde{X}_{i}\right)\right)=l\left(B_{i}\right)+s\left(B_{i}\right)-s$.

In Fig. 4 the numerical results are displayed for $T$ being a PLT or PVT, respectively, and for Cox processes $\left\{X_{n}\right\}$ with different values of $\kappa$, where we used the estimator introduced in Eq. 10. Furthermore, in Fig. 5 the results are shown which we obtained for network locations with a fixed distance $s$ to their HLC. For different values of $s$ and $\kappa$, we computed the density of the typical subtree length $v_{1}\left(T_{\text {sub }}^{*}\right)$ using difference quotients of the empirical distribution function $\widehat{F}(x ; n)$ given in Eq. 8 .

It can be seen that the shapes of all the densities presented in Figs. 4 and 5 are quite similar to each

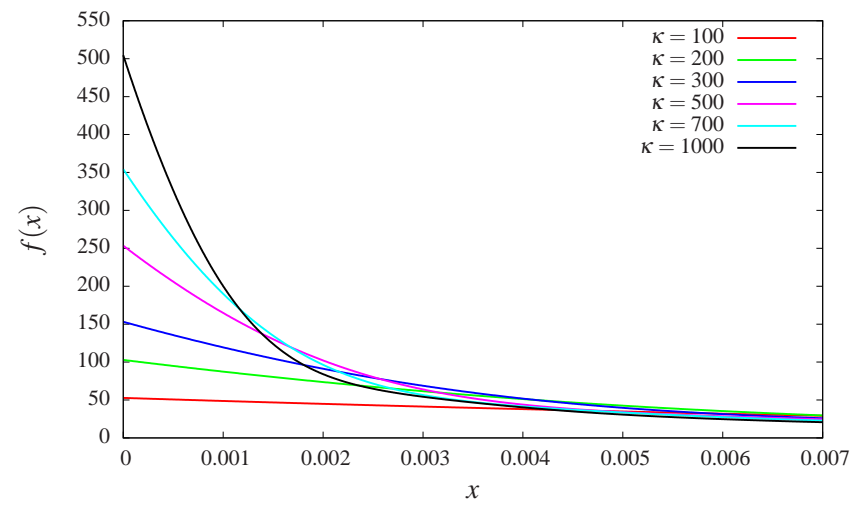

(b) PVT

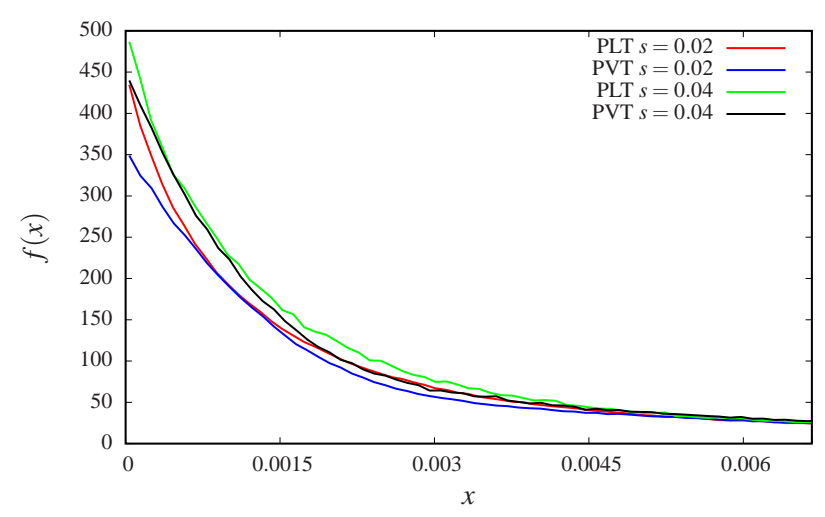

(b) $\kappa=700$

other. Furthermore, Fig. 5 shows that the density $f(x)$ of the typical subtree length $v_{1}\left(T_{\text {sub }}^{*}\right)$ does not depend too much on the particular choice of the (fixed) distance $s$ to the nearest HLC. On the other hand, the values of $f(x)$ change rather drastically if the model type of $T$ (PLT vs. PVT) or the scaling factor $\kappa$ is changed, see Figs. 4 and 5.

We also remark that knowing the density $f(x)$ of the typical subtree length $v_{1}\left(T_{\text {sub }}^{*}\right)$ and using the representation formula (Eq. 3 ) for the typical capacity $C^{*}$, we can compute, e.g., the expectation $\mathbb{E} h\left(C^{*}\right)$ for various functionals $h:[0, \infty) \rightarrow[0, \infty)$ of $C^{*}$, where

$$
\begin{aligned}
\mathbb{E} h\left(C^{*}\right) & =\mathbb{E} h\left(\sum_{i=1}^{K^{*}} C_{i}\right) \\
& =\sum_{k=0}^{\infty} \frac{1}{k !} \mathbb{E} h\left(\sum_{i=1}^{k} C_{i}\right) \int_{0}^{\infty} \mathrm{e}^{-\lambda_{\ell}^{\prime} x}\left(\lambda_{\ell}^{\prime} x\right)^{k} f(x) \mathrm{d} x,
\end{aligned}
$$

which is a consequence of Theorem 1. 


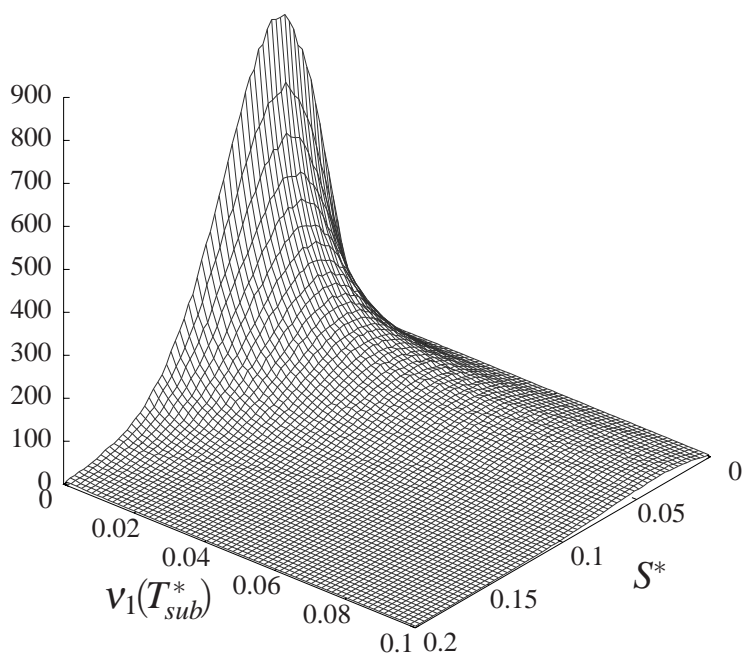

(a) PLT, $\kappa=100$

Fig. 6. Joint density of $\left(S^{*}, v_{1}\left(T_{\text {sub }}^{*}\right)\right)$

\section{DISCUSSION}

In this paper, the notion of capacity in the SSLM has been introduced. Furthermore, it has been shown how distributional characteristics of the typical capacity can be computed for two different types of network locations, where the algorithm is based on Monte Carlo simulation of the typical serving zone. Note that these results can be generalized in different ways, e.g., to typical capacities for further types of network locations. Possible examples are the nodes of the underlying tessellation or subfamilies of these nodes conditioning on the number of nodes passed on the shortest path to HLC. Another topic for future research could be the investigation of limit theorems regarding the distribution of typical capacity as $\kappa \rightarrow \infty$. Note that in Voss et al. (2009b) scaling limits of this type have been derived for the typical shortest path length $S^{*}$. It would be of great practical interest if such limit theorems can also be derived for the typical subtree length and the typical capacity, respectively. Furthermore, besides computing the densities of $S^{*}$ and $v_{1}\left(T_{\text {sub }}^{*}\right)$ separately, it is possible to determine the joint density of $S^{*}$ and $v_{1}\left(T_{\text {sub }}^{*}\right)$, seen from the perspective of the typical point of a Cox process on $T^{(1)}$, see Fig. 6 . From this two-dimensional density it is then possible to compute the density of $v_{1}\left(T_{\mathrm{sub}}^{*}\right)$ conditioning on $S^{*}=s$. Summarizing, the ideas which have been developed in the present paper can be combined with the fitting techniques for optimal network models

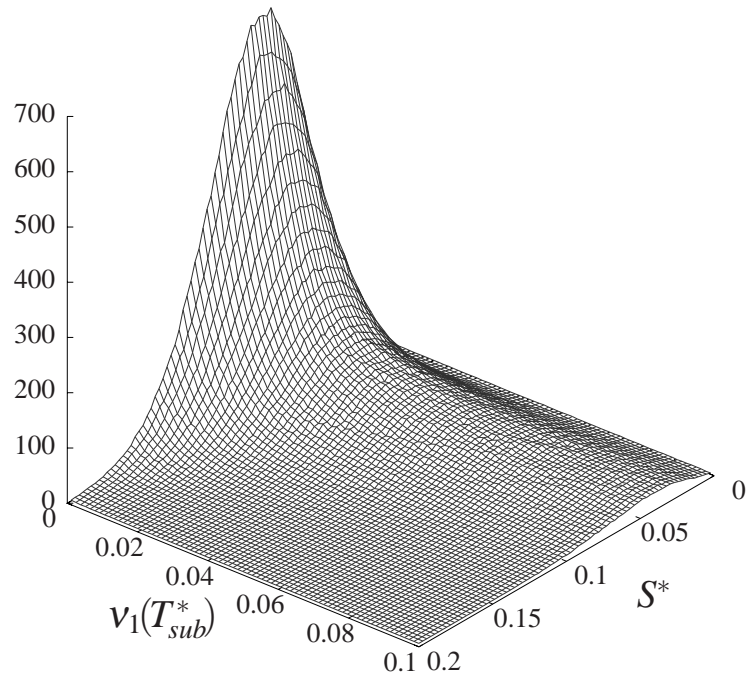

(b) PVT, $\kappa=100$

introduced in Gloaguen et al. (2006) in order to provide an efficient tool for capacity analysis in real (planned or existing) telecommunication networks.

\section{ACKNOWLEDGEMENTS}

This research was supported by Orange Labs through research grant 46143714 . The authors thank Steffen Müthing for his help in performing the computer experiments.

\section{REFERENCES}

Baccelli F, Błaszczyszyn B (2001). On a coverage process ranging from the Boolean model to the Poisson-Voronoi tessellation. Adv Appl Prob 33:293-323.

Baccelli F, Tchoumatchenko K, Zuyev S (2000). Markov paths on the Poisson-Delaunay graph with applications to routeing in mobile networks. Adv Appl Prob 32:118.

Baccelli F, Zuyev S (1996). Poisson-Voronoi spanning trees with applications to the optimization of communication networks. Operations Res 47:619-31.

Dijkstra, E (1959). A note on two problems in connexion with graphs. Numer Math 1:269-71.

Fleischer F, Gloaguen C, Schmidt V, Voss F (2009). Simulation of the typical Poisson-Voronoi-CoxVoronoi cell. J Stat Comput Sim 79:939-57.

Gloaguen C, Fleischer F, Schmidt H, Schmidt V (2005). Simulation of typical Cox-Voronoi cells, with a special 
regard to implementation tests. Math Meth Operations Res 62:357-73.

Gloaguen C, Fleischer F, Schmidt H, Schmidt V (2006). Fitting of stochastic telecommunication network models, via distance measures and Monte-Carlo tests. Telecommun Syst 31:353-77.

Gloaguen C, Fleischer F, Schmidt H, Schmidt V (2009a). Analysis of shortest paths and subscriber line lengths in telecommunication access networks. Netw Spat Econ DOI 10.1007/s11067-007-9021-z.

Gloaguen C, Voss F, Schmidt V (2009b). Parametric distance distributions for fixed access network analysis and planning. In: Proceedings of the $21^{\text {st }}$ International Teletraffic Congress, Paris, France (to appear).

Maier R, Mayer J, Schmidt V (2004). Distributional properties of the typical cell of stationary iterated tessellations. Math Meth Oper Res 59:287-302.

Neveu J (1976). Sur les mesures de palm de deux processus ponctuels stationnaires. Z Wahrscheinlichkeit 34:199203.
Schneider R, Weil W (2008). Stochastic and Integral Geometry. Berlin: Springer.

Stoyan D, Kendall WS, Mecke J (1995). Stochastic Geometry and its Applications. Chichester: J. Wiley \& Sons, $2^{\text {nd }}$ ed.

Voss F, Gloaguen C, Fleischer F, Schmidt V (2009a). Distributional properties of Euclidean distances in wireless networks involving road systems. IEEE J Sel Areas Commun 27:1047-55.

Voss F, Gloaguen C, Schmidt V (2009b). Scaling limits for shortest path lengths along the edges of stationary tessellations. Submitted. http://www.uni-ulm.de/fileadmin/website_uni_ulm/ mawi.inst.110/mitarbeiter/voss/Scaling_Limits.pdf.

Voss F, Gloaguen C, Fleischer F, Schmidt V (2009c). Density estimation of typical shortest path lengths in telecommunication networks. Submitted. http: //www.uni-ulm.de/fileadmin/website_uni_ulm/mawi. inst.110/mitarbeiter/voss/ShortestPath-18.09.09.pdf. 